\title{
Review: poor quality studies suggest that vitamin B-6 is beneficial in premenstrual syndrome
}

Wyatt KM, Dimmock PW,Jones PW, et al. Efficacy of vitamin B-6 in the treatment of premenstrual syndrome: systematic review. BMJ 1999 May 22;318:1375-81.

\author{
QUESTION: Is vitamin B-6 (pyridoxine) effective for relieving overall premenstrual \\ symptoms and depressive premenstrual symptoms?
}

\section{Data sources}

Studies were identified by searching Medline (1966-98), EMBASE/Excerpta Medica (1988-96), PsycLIT (197497), CINAHL (1982-97), and the Cochrane Controlled Trials Register with the terms premenstrual syndrome, pyridoxine, PMT, LLPDD, and PMDD. Bibliographies of relevant articles were scanned, and pharmaceutical companies were contacted.

\section{Study selection}

Published and unpublished studies in any language were selected if they were randomised, double blind, placebo controlled studies for which data could be acquired. Studies involving multivitamin supplements were included if the supplement contained $\geq 50 \mathrm{mg}$ of vitamin B-6. Authors were contacted if additional information was required.

\section{Data extraction}

Data were extracted on participants, dose and preparation of vitamin B-6, outcome measures, results, withdrawals, side effects, and quality scores. Main outcome was subjective improvement in overall premenstrual symptoms, and a secondary outcome was improvement in depressive premenstrual symptoms. High quality studies had scores $\geq 3$ on the Jadad scale (maximum 5 points) and $\geq 6$ on the authors' quality rating scale (maximum 8 points).

\section{Main results}

10 studies with 12 comparisons met the inclusion criteria. Sample sizes ranged from 31-434 patients (mean 98 patients). Doses ranged from $50 \mathrm{mg} /$ day (3 comparisons) to $600 \mathrm{mg} /$ day (1 comparison). Quality scores on the Jadad scale were 1 (3 studies), 2 (4 studies), and 3 (3 studies) points. On the authors' quality rating scale, scores were 3 (2 studies), 4 (5 studies), 5 (2 studies), and 6 (1 study) points. Quality scores were not used to exclude trials from the analysis because few high quality studies were found. Pooled results showed that vitamin B-6 relieved premenstrual symptoms (combined odds ratio [OR] 1.57, 95\% CI 1.40 to 1.77). The inclusion of 1 study in the meta-analysis led to statistically significant heterogeneity $(\mathrm{p}<0.001)$. When this study was removed from the pooled result, the combined OR was 2.32 (CI 1.95 to 2.54). 5 trials reported data on premenstrual depressive symptoms and showed a benefit for vitamin B-6 (combined OR 2.12, CI 1.80 to 2.48). 1 study led to heterogeneity $(p<0.001)$ and the removal of this study resulted in a combined OR of 1.69 (CI 1.39 to 2.06). The 3 high quality studies according to the Jadad scale showed a benefit for vitamin B-6, whereas the 1 high quality study according to the authors' quality rating showed no effect; this study had low power for detecting any differences. No dose response was found in the 9 trials.

\section{Conclusions}

A meta-analysis of randomised, double blind, placebo controlled trials shows that vitamin B-6 relieves overall premenstrual and depressive premenstrual symptoms. Most studies were of poor quality.

\section{COMMENTARY}

Epidemiological surveys have estimated that as many as $75 \%$ of women with regular menstrual cycles experience some physical and psychological symptoms premenstrually. ${ }^{1}$ Vitamin B-6 is one of the numerous treatments suggested for premenstrual syndrome (PMS). Wyatt et al have done a systematic review of trials comparing the efficacy of vitamin B-6 with placebo in women with PMS. Although their metaanalysis includes a few more trials than a previous review published in $1991,{ }^{2}$ they too conclude that "there is insufficient evidence of high enough quality to give a confident recommendation for using vitamin B-6 in the treatment of premenstrual syndrome" ( $\mathrm{p}$ 1378). Wyatt et al used extensive data sources and rigorous selection criteria to identify 10 relevant trials. They used 2 different scales to evaluate the methodological quality of each trial. 9 trials (after excluding 1 trial that caused statistical heterogeneity) were included in the meta-analysis, although only 4 were rated as high quality studies on either of the 2 scales. The 9 trials differed in many ways including the outcomes, the dosage of vitamin B-6, and whether the intervention was vitamin B-6 alone or a multivitamin. Moreover, the authors did not consider the severity of premenstrual symptoms. This is an important variable because women who meet criteria for premenstrual dysphoric disorder, the more severe form of PMS, usually do not respond to more conservative interventions, such as vitamin B-6. ${ }^{3}$ The lack of a dose response adds to the uncertainty about the effectiveness of vitamin B-6 for PMS.

This review highlights the need for well designed trials to compare vitamin B-6 with placebo to establish more accurate recommendations for treatment. In the meantime, what do clinicians tell patients? Given that vitamin B-6 is readily available, relatively inexpensive, and generally has minimal side effects in doses $<200 \mathrm{mg}$, clinicians may suggest that patients take $\leqslant 100 \mathrm{mg}$ /day as a conservative treatment option before considering drugs with stronger evidence of effectiveness such as selective serotonin reuptake inhibitors.

Mary Macdougall, RN, MHSc

Research Coordinator, Women's Health Concerns Clinic St Joseph's Hospital Hamilton, Ontario, Canada

1 Steiner M, Yonkers KA. Depression in women. UK: Martin Dunitz Ltd;1998:2.

2 Kleijnen J, Ter Riet G, Knipschild P. Vitamin B-6 in the treatment of the premenstrual syndrome-a review. $\mathrm{Br} \mathrm{J} \mathrm{Obstet}$ Gynaecol 1990;97:847-52.

3 Steiner M. Premenstrual syndromes. Annu Rev Med 1997;48:447-55 